\title{
Chronic and Severe Non-Lichenoid Oral Ulcers Induced by Nivolumab - Diagnostic and Therapeutic Challenge: A Case Report
}

\author{
Andrés F. Cardona ${ }^{a-c} \quad$ Alejandro Ruiz-Patiñob,c Luisa Ricaurte ${ }^{b, c}$ \\ Zyanya Lucia Zatarain-Barrón ${ }^{d}$ Feliciano Barrón ${ }^{d}$ Oscar Arrietad \\ ${ }^{a}$ Clinical and Translational Oncology Group, Clinica del Country, Bogotá, Colombia; \\ bFoundation for Clinical and Applied Cancer Research - FICMAC, Bogotá, Colombia; \\ 'Molecular Oncology and Biology Systems Research Group (Fox-G), Universidad El Bosque, \\ Bogotá, Colombia; ${ }^{d}$ Thoracic Oncology Unit, National Cancer Institute (INCan), \\ Mexico City, Mexico
}

\author{
Keywords \\ Head and neck cancer · Oral ulcer · Immunotherapy · Toxicity · Microbiome analysis
}

\section{Abstract}

Due to the widespread use of immune checkpoint inhibitors and the growing research efforts in this area, immune-mediated toxicity is well recognized. Nonetheless, few severe cases of oral or upper gastrointestinal tract mucosal involvement have been documented. Early recognition and prompt treatment are key to the adequate management of these patients. We present a male 93-year-old patient with an advanced head and neck tumor treated with nivolumab who developed severe oral ulcers. After discontinuation of nivolumab, he received initial steroid treatment without any significant improvement. Histopathologic analysis of the lesions revealed a pattern similar to graft versus host disease. Extrapolating the results of colchicine mouth washing in patients with active oral ulcers and Behçet's disease, this strategy was implemented with concomitant metronomic cyclophosphamide, achieving complete ulcer resolution. Metagenomic oral bacterial sequencing during instauration of the lesions and highest extension revealed a significant decrease in microbiomic diversity and expansion of Haemophilus parainfluenzae similar to patients with active Behçet's disease. In conclusion, oral ulcers associated with immune checkpoint inhibitors correspond to a difficult-to-treat entity that could physiopathologically be related to both graft versus host disease and Behçet's disease. 
Cardona et al.: Oral Ulcers Induced by Nivolumab

\section{Introduction}

There is a wide spectrum of dermatologic toxicities associated with immune checkpoint inhibitors (ICIs) including anti-CTLA-4 and anti-PD-1/PD-L1 antibodies. Dermatologic toxicities (any type and any grade) are twice as frequent in patients who receive anti-CTLA-4 antibody therapy compared to those who receive anti-PD-1/PD-L1 antibodies, i.e., 68 versus $\sim 38 \%$, respectively. The most common dermatologic toxicities can be categorized into lesions that are inflammatory, immunobullous, alterations of epidermal keratinocytes, and alterations of epidermal melanocytes [1]. Besides skin reactions, ICIs may also induce oral mucosal injuries. Oral lichenoid reactions with reticulated white streaks and papular, plaque-like, ulcerative, or atrophic/erythematous lesions in the presence of anti-PD-1/PD-L1 agents have sporadically been described [2]. Patients usually remain asymptomatic and lesions appear to be reversible after topical corticosteroid treatment with no need to suspend the current immunotherapy regimen $[3,4]$. Histologic analysis of some ICI-derived oral ulcers displayed a patchy and/or florid lichenoid interface in the upper lamina propria and predominantly CD4/CD8-positive band-like $\mathrm{T}$ cell infiltrate. This condition is occasionally associated with xerostomia $(\approx 6 \%)$, dysgeusia and esophagitis $[5,6]$.

Anti-PD-1 therapy has received a multinational approval for the treatment of metastatic melanoma, classical Hodgkin lymphoma (relapsed or refractory), unresectable or metastatic microsatellite instability-high cancer, non-small cell lung cancer, advanced or metastatic urothelial carcinoma and metastatic or recurrent head and neck squamous cell cancer after failure of a platinum-containing regimen. This last indication was given based on promising results of two phase II studies and confirmatory large phase III trials [7, 8].

Due to the widespread use of ICIs and the growing research efforts in this area, immune mediated toxicity is well recognized. Nonetheless, few severe cases of oral or upper gastrointestinal tract mucosal involvement have been documented. Early recognition and prompt treatment are key to the adequate management of these patients. In this case report, we present the case of a patient who developed severe oral ulcers that were refractory to steroidal support. We also developed a few hypotheses regarding the pathologic findings and implications of the microbiome environment seen in this patient. From a therapeutic point of view, a strategy based on the treatment for both Behçet's disease and graft versus host disease is described.

\section{Case Presentation}

A 93-year-old male physician with a history of deep vein thrombosis, hypertension, atrial fibrillation, chronic moderate sarcopenia and mild hypoacusis was diagnosed with $\mathrm{T}_{2} \mathrm{~N}_{1} \mathrm{M} 0$ squamous cell carcinoma of the pharynx with partial involvement of the base of the tongue. Due to comorbid conditions and the patient's rejection of total glossopharyngectomy, tracheostomy and cervical lymphadenectomy, hypofractionated radiotherapy (40 Gy) plus cetuximab (loading dose of $400 \mathrm{mg} / \mathrm{m}^{2} 5-7$ days before radiotherapy initiation, followed by cetuximab $250 \mathrm{mg} / \mathrm{m}^{2}$ weekly for 5 doses, total $1,650 \mathrm{mg} / \mathrm{m}^{2}$ ) was administered under nutritional and metabolic support. A partial response with good tolerance was achieved. Local progression occurred within 12.4 months after the end of initial therapy. Two months later, the patient received nivolumab $200 \mathrm{mg}$ i.v. every 15 days. A complete response after 4 months of initiation was observed (Fig. 1). During therapy, the patient developed asymptomatic primary hypothyroidism. There was evidence of positive serum anti-thyroid peroxidase (TSH $9.44 \mathrm{mIU} / \mathrm{L}$, normal value $0.30-5.50$; T3 $3.1 \mathrm{pmol} / \mathrm{L}$, normal value 3.5-6.0; and T4 7.9 pmol/L, normal value 10.0-23.0). Steroidal support was not used due to basal sarcopenia. The patient was started on levothyroxine $50 \mu \mathrm{g}$ per day with optimal results. 


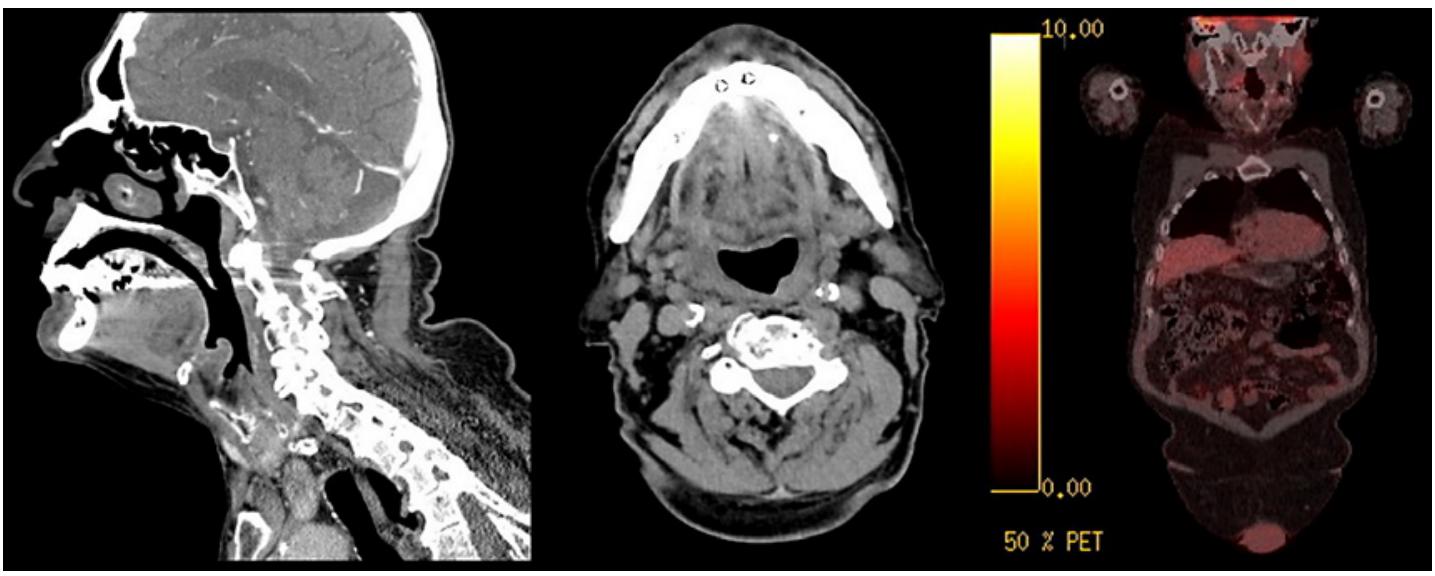

Fig. 1. Complete response of the right supraglottic and pharyngeal lesion, with previous compromise on the vallecula, aryepiglottic fold, and right side of the epiglottis after treatment with intensity-modulated radiotherapy plus cetuximab.
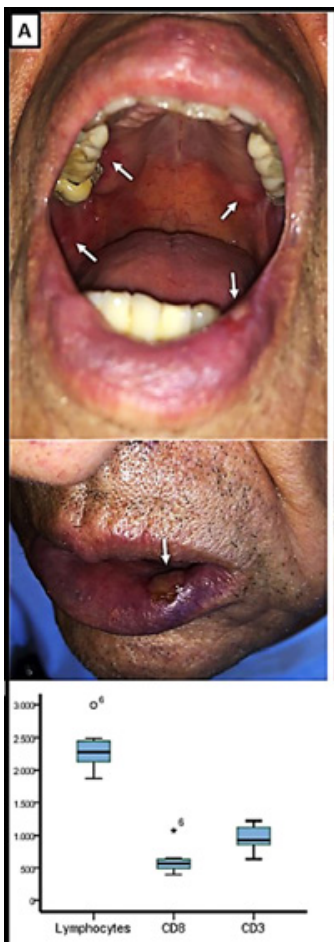
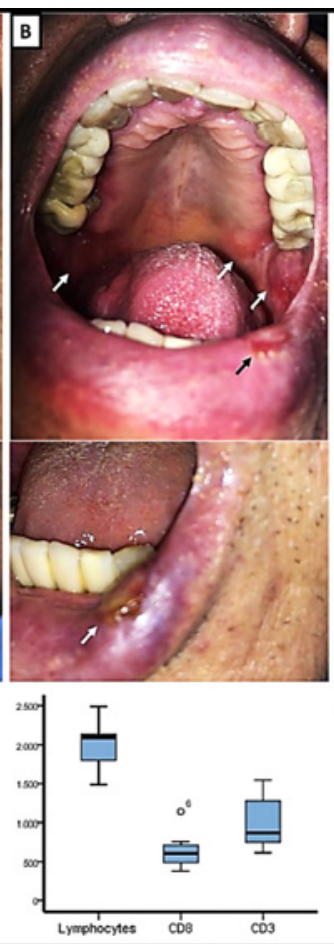
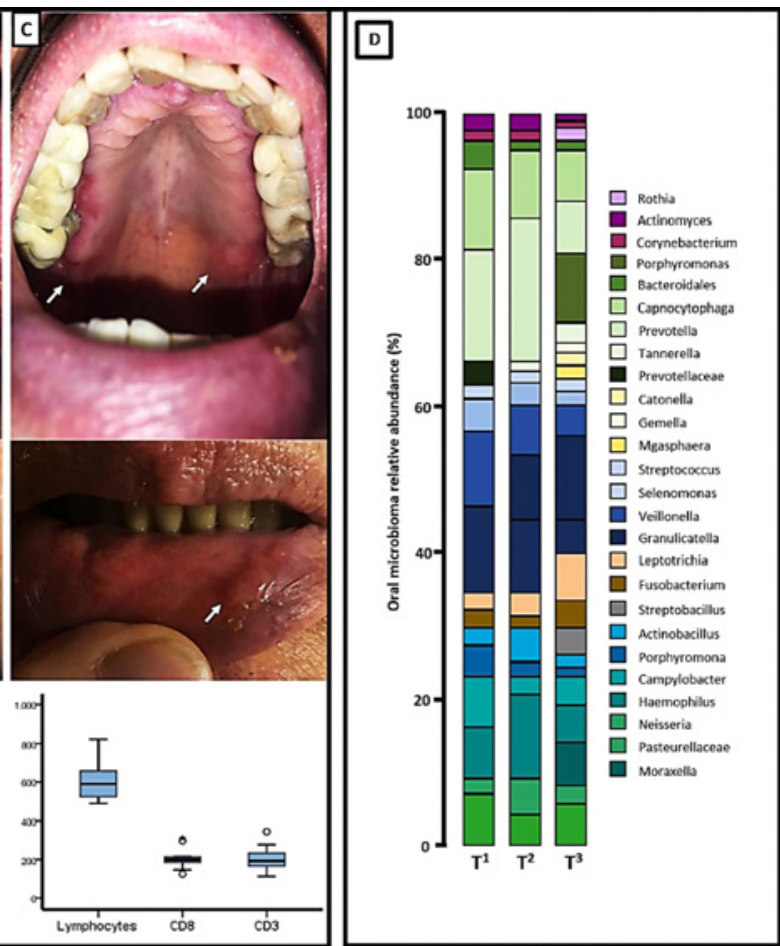

Fig. 2. Oral lesions, leukocyte counts and subpopulations at the start of symptoms (A), after administration of etoposide (B), and after systemic treatment with metronomic cyclophosphamide and oral rinse with colchicine (C). D Microbiome diversity study at the same time points.

After 10 cycles, the patient reported severe odynophagia. Multiple erythematous ulcers in the buccal mucosa and tongue were seen. The lesions rapidly progressed, the inferior lip (with recurrent bleeding) and almost $50 \%$ of the mucosal membrane were compromised (Fig. 2A-C). There was no fever, dysphagia, diarrhea or test evidence of herpes virus, mycotic, Epstein-Barr virus or cytomegalovirus infection. It was decided to completely stop treatment with nivolumab. 


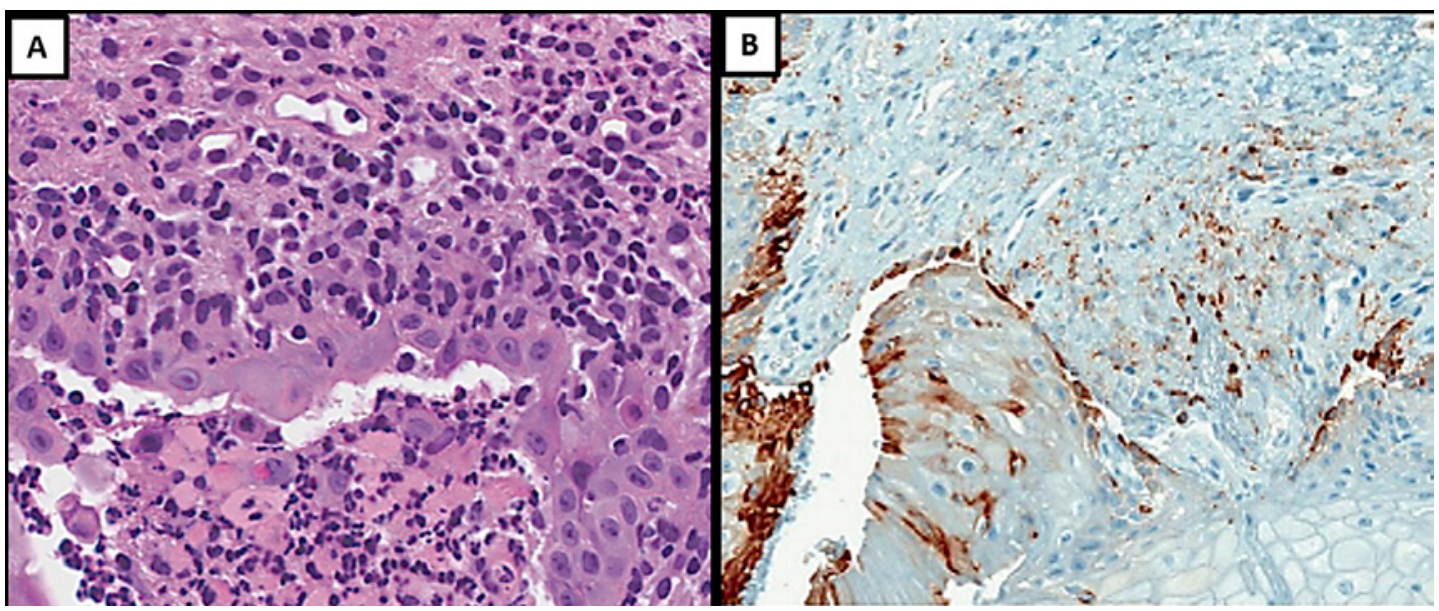

Fig. 3. A Oral ulcer contained and surrounded by polymorphonuclear cells and confined cell debris; outside this zone and extending diffusely throughout the lamina propria, a large number of infiltrating lymphocytes and macrophage-like cells were seen $(\times 40)$. B Inflammatory cell infiltrate (CD8+ stained; antibody CONFIRM anti-CD8, SP57) associated with oral mucosa ulceration $(\times 10)$.

He was started on sucralfate, magic mouth wash (MuGard ${ }^{\mathrm{TM}}$, oral mucoadhesive) and a 14-day course of oral prednisone ( $1 \mathrm{mg} / \mathrm{kg}$ every day) and topical triamcinolone acetonide; however, these treatments had a minimal response. Two weeks later, the patient was admitted to the hospital due to progressive dysphagia, severe pain and rapid weight loss $(\approx 8 \mathrm{~kg})$. Initial blood tests showed a normal white blood cell count of $8.4 \times 10^{9} / \mathrm{L}$ (neutrophils $6.87 \times 10^{9} / \mathrm{L}$, lymphocytes $0.940 \times 10^{9} / \mathrm{L}$, eosinophils $0.5 \times 10^{9} / \mathrm{L}$ ) and platelets at $296,000 \times 10^{9} / \mathrm{L}$. Intravenous methylprednisolone $2 \mathrm{mg} / \mathrm{kg} /$ day was started without improvement and increased pain. Gastroscopy did not reveal signs of esophagitis in the presence of mild antral gastritis. Due to refractory behavior of the oral ulcers and based on the histologic findings (Fig. 3A, B) a chronic graft-versus-host disease (GVHD) reaction was considered. Based on a recent description by Rios et al. [9], oral etoposide $25 \mathrm{mg}$ p.o. every $12 \mathrm{~h}$ for 7 days was administered. Transitory pain improvement without any changes in the ulcer distribution was noted (Fig. 2B). Because of the nutritional state and the limitations with food ingestion, parenteral nutrition was administered, and gastrostomy tube was considered as an option.

It was hypothesized that the mouth ulcers were caused by an exaggerated and pathologic immune system reactivity against the oral mucosa. Based on this, oral cyclophosphamide was initiated in a metronomic schedule: $50 \mathrm{mg}$ every day for 21 days in a regimen of 28 days for 2 cycles.

Additionally, and extrapolating the benefit of periodical mouth washing with colchicine observed in chronic and recurrent ulcers in Behçet's disease [10], treatment was initiated with a solution of $1 \mathrm{mg}$ in $150 \mathrm{~mL}$ every $8 \mathrm{~h}$. The patient's blood lymphocyte count was measured and divided into CD8+ and CD3+ populations at different time points (at the start of symptoms, $\mathrm{t}^{1}$; after the administration of etoposide, $\mathrm{t}^{2}$; after systemic treatment with metronomic cyclophosphamide and oral rinse with colchicine, $\mathrm{t}^{3}$ ) (Fig. 2A-C). Shortly after the beginning of treatment, an almost complete remission of the lesions and optimal pain control was achieved (Fig. 2C).

Simultaneously, metagenomic evaluation of oral microbiome was also performed (at time points $\mathrm{t}^{1}, \mathrm{t}^{2}$ and $\mathrm{t}^{3}$ ). We used a high-throughput sequencing of bacterial 16S rRNA (Fig. 2D) approach as follows: sequencing of 16S rRNA gene amplicon DNA from patient saliva samples $\left(\mathrm{T}^{1}, \mathrm{~T}^{2}\right.$ and $\left.\mathrm{T}^{3}\right)$ was subjected to amplification of polymerase chain reaction (PCR) 
using primers directed at hypervariable regions 3-4 (V3-V4) of the $16 \mathrm{~S}$ rRNA gene (341F and 806R). The PCR products were quantified using Qubit (Invitrogen, Carlsbad, CA, USA), multiplexed at an even concentration and subject to 300-bp pair-end sequencing on Illumina MiSeq platform (Illumina, Inc., San Diego, CA, USA). The resulting raw reads were filtered using a quality control criterion (minimum average quality score 20; maximum number of ambiguous $\mathrm{N}$ base 3) and matched to sequences spanning the entire V3-V4 amplicon using PANDAseq [11]. A total of 623,567 merged sequences were generated from the 3 samples. Merged sequences with $97 \%$ nucleotide sequence identity were binned into operational taxonomic units using UPARSE [12]. Based on the RDP classifier, a representative sequence of each operational taxonomic unit was assigned to a taxonomic level in the RDP database using 0.8 as the minimum confidence threshold [11]. The OTU was rarefied to 7,615 sequences/sample. Alpha and beta diversity were calculated using QIIME with the default parameters. Following the DNA Sample Prep v2 Guide (Illumina, Inc., San Diego, CA, USA), we constructed the DNA paired-end libraries with an insert size of $500 \mathrm{bp}$ for the samples.

A significant reduction in bacterial diversity was observed throughout the time points. The bacterial species during the periods $\mathrm{t}^{1-3}$ most commonly found were Prevotella melaninogenica, Veillonella dispar, Porphyromona gingivalis, Granulicatella and Leptotrichia. At $\mathrm{t}^{2}$, an enrichment in the concentration of P. melaninogenica and Haemophilus parainfluenzae was noted. At 3 months of follow-up, the patient maintains an oncologic complete response with no evidence of new ulcers or other ICI-derived complications.

\section{Discussion and Conclusions}

The present case revealed through a histopathologically proven approach the chronic and steroid refractory destruction of the mucosal membrane present in the patient's mucosal ulcers. Due to the extreme rareness of this phenomenon, few publications regarding this complication are available. One of them is a study by Namiki et al. [13] conducted in an 84-year-old woman treated with nivolumab because of similar oral lesions. Immunohistochemical findings revealed a direct CD8+ T lymphocyte-mediated mucosal destruction and a decreased number of CD1a-positive dendritic cells in the epidermis, suggesting a reaction similar to the one described in GVHD. Furthermore, CD8-positive T lymphocytes infiltrating the oral mucosa revealed PD-1 expression. These findings suggest that the administration of an anti-PD-1 agent alters the immune system and generates cross-reactivity against several antigens present in the oral epithelium. Although alterations of the oral and esophageal mucosa have been reported, in association with anti-PD-1 agents, these findings have been estimated to be around $1 \%$ for nivolumab and $2 \%$ for pembrolizumab. Only nonsevere reactions have been documented. Furthermore, histopathologic findings of these lesions are also scarce [14].

This case offers two possible hypotheses, both with therapeutic implications. GVHD of the mucosa is a disease that is frequently observed in allogeneic bone marrow recipients. It is usually managed with systemic corticosteroids as a first line. The setting of mucosal involvement is particularly challenging with the majority of cases being refractory. Topical corticosteroids have been tested; most patients exhibit either no response or a partial response [15]. The oral ulcers experienced by the patient discussed in the present work were also refractory to systemic therapy. On the other hand, and similarly to what is observed in Behçet's disease, oral colchicine proved to be highly effective in the treatment of the patient's ulcers. This could potentially suggest that this phenomenon is influenced by a similar vasculitis pathogenesis. Contrary to Behçet's disease, evidence of improvement of oral ulcers seen in GVHD with colchicine is lacking. Furthermore, metagenomic bacterial classification of the 
Cardona et al.: Oral Ulcers Induced by Nivolumab

mucosa revealed that the most enriched taxa during the largest ulcer extension accounted for H. parainfluenzae and that this enrichment was reduced after the resolution of the lesions. Moreover, a reduction in colony diversity was observed. These findings are concordant with patients suffering from active Behçet's ulcers compared to healthy controls and could potentially suggest either a causative role or a consequence of the lesion's occurrence [16].

In conclusion, treatment of oral lesions as an adverse reaction to ICIs is a therapeutic challenge. Given the low incidence and lack of robust evidence of their management, individualized treatment and translational approaches could offer relief for some patients. Further studies exploring wider aspects of microbiome relevance are also warranted.

\section{Statement of Ethics}

No personal information or images that could identify the patient were used. Additionally, and according to local regulations, no psychologic, physiologic, biologic or societal variables were modified, therefore making the study a risk-free study and therefore it was not required to be approved by an institutional ethics board. Although no personal information was included, an informed consent for publication was obtained.

\section{Disclosure Statement}

The authors declare no relevant conflicts of interest.

\section{Funding Sources}

This study did not require funding.

\section{Author Contributions}

A.F.C. and A.R.-P.: Collection of data, analysis, draft and approval of the manuscript. L.R., Z.L.Z.-B., F.B., and O.A.: Analysis of data, draft and approval of the manuscript.

\section{References}

1 Curry JL, Tetzlaff MT, Nagarajan P, Drucker C, Diab A, Hymes SR, et al. Diverse types of dermatologic toxicities from immune checkpoint blockade therapy. J Cutan Pathol. 2017 Feb;44(2):158-76.

2 Lacouture M, Sibaud V. Toxic side effects of targeted therapies and immunotherapies affecting the skin, oral mucosa, hair, and nails. Am J Clin Dermatol. 2018 Nov;19(Suppl 1):31-9.

3 Shi VJ, Rodic N, Gettinger S, Leventhal JS, Neckman JP, Girardi M, et al. Clinical and histologic features of lichenoid mucocutaneous eruptions due to anti-programmed cell death 1 and anti-programmed cell death ligand 1 immunotherapy. JAMA Dermatol. 2016 01;152(10):1128-36.

4 Sibaud V, Eid C, Belum VR, Combemale P, Barres B, Lamant L, et al. Oral lichenoid reactions associated with anti-PD-1/PD-L1 therapies: clinicopathological findings. J Eur Acad Dermatol Venereol. 2017 Oct;31(10): e464-9.

5 Boike J, Dejulio T. Severe esophagitis and gastritis from nivolumab therapy. ACG Case Rep J. 2017;4:e57.

6 Vigarios E, Epstein JB, Sibaud V. Oral mucosal changes induced by anticancer targeted therapies and immune checkpoint inhibitors. Support Care Cancer. 2017;25(5):1713-39.

7 Bauml J, Seiwert TY, Pfister DG, Worden F, Liu SV, Gilbert J, et al. Pembrolizumab for platinum- and cetuximabrefractory head and neck cancer: results from a single-arm, phase II study. J Clin Oncol. 2017 May;35(14): 1542-9. 
8 Seiwert TY, Burtness B, Mehra R, Weiss J, Berger R, Eder JP, et al. Safety and clinical activity of pembrolizumab for treatment of recurrent or metastatic squamous cell carcinoma of the head and neck (KEYNOTE-012): an open-label, multicentre, phase 1b trial. Lancet Oncol. 2016 Jul;17(7):956-65.

9 Rios A, Cen P, Dinh B, Mays SR, Patel AB. Dramatic response of nivolumab-associated psoriasiform dermatitis to etoposide. Eur J Cancer. 2019 Jan;107:97-9.

10 Saleh Z, Arayssi T. Update on the therapy of Behçet disease. Ther Adv Chronic Dis. 2014 May;5(3):112-34.

11 Masella AP, Bartram AK, Truszkowski JM, Brown DG, Neufeld JD. PANDAseq: paired-end assembler for illumina sequences. BMC Bioinformatics. 2012;13:31.

12 Edgar RC. UPARSE: highly accurate OTU sequences from microbial amplicon reads. Nat. Methods. 2013; 10(10):996-8.

13 Namiki T, Hanafusa T, Ueno M, Miura K, Yokozeki H. Severe oral ulcers associated with nivolumab treatment. JAMA Dermatol. 2017 Feb;153(2):235-7.

14 Acero Brand FZ, Suter N, Adam J-P, Faulques B, Maietta A, Soulières D, et al. Severe immune mucositis and esophagitis in metastatic squamous carcinoma of the larynx associated with pembrolizumab. J Immunother Cancer. 2018 Mar;6(1):22.

15 Noce CW, Gomes A, Shcaira V, Corrêa MEP, Moreira MCR, Silva Júnior A, et al. Randomized double-blind clinical trial comparing clobetasol and dexamethasone for the topical treatment of symptomatic oral chronic graft-versus-host disease. Biol Blood Marrow Transpl. 2014 Aug;20(8):1163-8.

16 Coit P, Mumcu G, Ture-Ozdemir F, Unal AU, Alpar U, Bostanci N, et al. Sequencing of $16 \mathrm{~S}$ rRNA reveals a distinct salivary microbiome signature in Behçet's disease. Clin Immunol. 2016;169:28-35. 